\title{
Antibody opsonization of tumor cell membranes using hapten-PEG-lipid conjugates
}

\author{
Tosihiko Sugiura ${ }^{1}$, Koichi Kato $^{1}$, Teruyuki Nagamune ${ }^{1,2,3}$ \\ 1. Department of Chemistry and Biotechnology, University of Tokyo, Tokyo, Japan. 2. Department of Bioengineering, \\ Graduate School of Engineering, University of Tokyo, Tokyo, Japan. 3. Center for NanoBio Integration, University of \\ Tokyo, Tokyo, Japan.
}

Correspondence: Prof. Teruyuki Nagamune. Address: Department of Chemistry and Biotechnology, Graduate School of Engineering, University of Tokyo, 7-3-1 Hongo, Bunkyo-ku, Tokyo 113-8656, Japan.

Email: nagamune@bio.t.u-tokyo.ac.jp

Received: August 31, 2015

Accepted: November 9, $2015 \quad$ Online Published: November 16, 2015

DOI : $10.5430 /$ jbei.v2n2p1

URL: http://dx.doi.org/10.5430/jbei.v2n2p1

\begin{abstract}
Dendritic cell (DC) immunotherapy is a newly developed type of tumor immunotherapy. As a method for generating antigen-presenting DCs, the phagocytosis of whole tumor cells (WTCs) opsonized with antibodies by DCs (antibody-dependent cellular phagocytosis, ADCP) is especially efficient. To opsonize tumor cells, tumor-specific antibodies are usually required. Therefore conventional ADCP was limited to tumor types for which antigens have been identified and antigen-specific antibodies are available. Herein, we developed a general method to promote ADCP by DCs using an arbitrary antibody and evaluated its antitumor effects. To opsonize tumor cells with a tumor-nonspecific antibody, we modified tumor cell surfaces with PEG-lipid-hapten conjugates and a conjugated anti-hapten antibody. By co-culturing DCs with the antibody-opsonized tumor cells, the phagocytosis and DC maturation rates were enhanced by $\sim 20 \%$ and $\sim 10 \%$, respectively, compared with co-cultured non-opsonized tumor cells. The administration of tumor antigen-loaded matured DCs to mice resulted in enhanced tumor rejection and long-term survival. The advantages of this method are that tumor-specific antibodies are not required and DCs can present uncharacterized antigens by phagocytosing WTCs and associated antigens. Therefore, this method might be applicable to various types of tumors for which specific antigens have not yet been identified.
\end{abstract}

\section{Key words}

Antibody-dependent cellular phagocytosis, Cell surface engineering, Dendritic cell immunotherapy, Tumorimmunotherapy

\section{I ntroduction}

Since the discovery of tumor-specific cytotoxic T lymphocytes (CTLs), tumor immunotherapy has received intense clinical interest. In CTL-mediated immunotherapy, the efficient induction of strong tumor-specific CTLs from CD8 ${ }^{+} \mathrm{T}$ cells is required for the ultimate endpoints of most cancer immunotherapy regimens. Earlier attempts to induce CTLs in vivo, however, did not achieve sufficient clinical results. Therefore, there is great interest in using professional antigen-presenting cell-based immunotherapies for tumor vaccination. Notably, dendritic cells (DCs), which play a pivotal 
role in initiating systemic immune responses, including helper $\mathrm{T}(\mathrm{Th})$ cells, natural killer (NK) cells, and CTLs, are receiving attention as a therapeutic cell type ${ }^{[1-4]}$. Furthermore, combinations of DC immunotherapy with targeted therapies toward molecular pathways that promote tumour growth and maintenance also represent potentially promising strategies to improve clinical outcomes for solid tumors ${ }^{[3,5]}$.

Immature DCs are generated from patient progenitor cells, and antigen-presenting mature DCs are prepared by loading with tumor-antigens and stimulation by cytokines (see Figure 1). These mature antigen-presenting DCs are administered to the same donor patient and can activate $\mathrm{CD}^{+}$and $\mathrm{CD}^{+} \mathrm{T}$ cells. These events occur via several simultaneous interactions between cell surface molecules, such as T cell receptor-tumor antigen-bound MHC class I or II, CD8-MHC class I, CD4-MHC class II, and CD28-CD86, molecules that are expressed on the surfaces of T cells or antigen-presenting mature DCs, respectively. Tumor-specific CTLs are generated from activated CD $8^{+} \mathrm{T}$ cells that undergo clonal expansion if they receive IL-2 stimulation (i.e. a "help" signal). This induces the proliferation of CTLs specific for the target tumor antigen that can then travel throughout the body to search for antigen-positive tumor cells. Upon encountering tumor cells, CTLs release the cytotoxic molecule perforin, which forms pores in the target tumor cell plasma membrane to facilitate granzyme entry into tumor cells, which then activates the caspase cascade and eventually initiates apoptosis.

Figure 1. A schematic diagram of DC immunotherapy for tumor vaccination

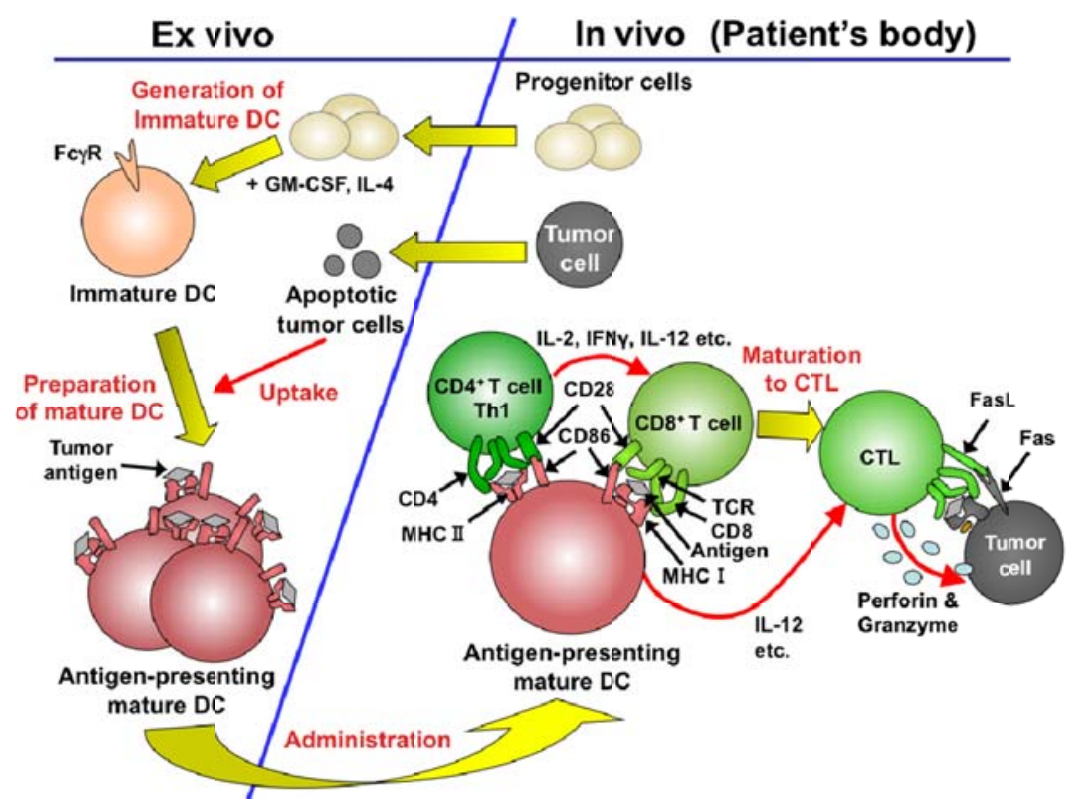

An important issue in DC therapy is the effective preparation of mature antigen-presenting DCs ex vivo. There are several methods for preparing mature DCs, such as transfection of immature DCs with tumor-antigen DNA or mRNA molecules ${ }^{[6]}$,to facilitate immature DC uptake of tumor-antigen peptides ${ }^{[7]}$, antigen-IgG immune complexes ${ }^{[8-10]}$ or whole tumor cell (WTC) lysates ${ }^{[11]}$, fusion of immature DCs with tumor cells ${ }^{[12-14]}$, or the uptake of WTCs by immature DCs through receptors on the DC cell surface ${ }^{[15,16]}$. Among these antigen sources, WTCs might be useful because they do not assume prior knowledge of the sequence of the antigenic peptides or necessitate human leukocyte antigen analysis of patients ${ }^{[3,11,16]}$.

DC immunotherapy using WTCs as tumor antigen sources may have some possibility that unknown number of autoantigens from tumor cells are also presented by mature DCs, resulting in interference in original immunity and generation of autoimmune diseases. However it has been reported that "vaccination of mature DCs is safe (eliciting no autoimmunity or other adverse effects) and has led the induction of melanoma-specific T-cell immunity" ${ }^{[3]}$. Furthermore, administering mature DCs loaded with WTCs can stimulate a strong polyclonal T cell immunity against numerous tumor antigens derived from WTCs, which can overcome some of the limitations of using single tumor-associated antigens, such as the potential for immune escape variants or the absence of $\mathrm{CD}^{+}$Th cell epitopes ${ }^{[11]}$. This advantage could compensate 
for autoimmune disadvantage. Accordingly, mature DCs loaded with WTC antigens have been used for immunizations in animal models and shown to induce both prophylactic and protective antitumor immunity in vivo ${ }^{[12,14,17-20]}$.

For the receptor-mediated internalization of WTCs, the phagocytosis of antibody-opsonized WTCs (antibody-dependent cellular phagocytosis, ADCP) by immature DCs through Fc receptor ( $\mathrm{Fc} \gamma \mathrm{R})$ is highly efficient ${ }^{[21-24]}$. Binding of the $\mathrm{Fc}$ domain of IgG to Fc $\gamma R$ on immature DCs promotes both the phagocytosis of immature DCs and the presentation of antigens derived from WTCs to MHC receptors on the DC cell surface. Specifically, DCs can efficiently present tumor cell antigens, which are phagocytized through a Fc $\gamma$ Rs-mediated pathway, on both MHC I and II. Subsequently, antigen-loaded DCs can activate both CTLs and NK cells ${ }^{[16,23]}$. Therefore, ADCP can induce effective antitumor responses by mature DCs.

To opsonize tumor cells, however, tumor-specific antibodies are usually required ${ }^{[21,22,24]}$. Therefore, conventional ADCP is only possible when the antigens are identified for a type of tumor and antigen-specific antibodies are available. We have developed reagents for rapidly anchoring various molecules to cell membranes (i.e., a biocompatible anchor for membrane, BAM) ${ }^{[25-27]}$. Recently, we successfully opsonized tumor cells with an arbitrary antibody-BAM conjugate and enhanced the phagocytosis rate of WTCs by immature DCs two-fold ${ }^{[28]}$. Although optimization of the chemical conjugation conditions of BAM to antibody minimized interference with the interaction between the Fc domain of IgG on tumor cells and the Fc $\gamma \mathrm{R}$ on immature DCs, it is difficult to control antibody-conjugation sites. This can result in insufficient spatial control of $\mathrm{Fc}$ domain orientation toward Fc $\gamma \mathrm{R}$ on immature DCs. We also successfully opsonized tumor cells with an antibody Fc domain-BAM conjugate, in which a BAM was site-specifically fused to the end of the Fc domain C-terminus by sortase A-mediated enzymatic ligation, thereby enhancing the phagocytic rate of WTCs by immature DCs four-fold ${ }^{[29]}$. However, the production yield of soluble intact $\mathrm{Fc}$ domains in mammalian cell culture and that of $\mathrm{Fc}$ domain-BAM conjugates prepared by sortase A-mediated ligation reactions might be insufficient for use in further clinical applications.

Herein, we aimed to develop a more general method to promote ADCP by DCs using an arbitrary antibody. To more precisely control the spatial orientation of the antibody Fc domain, we first modified tumor cell surfaces with a BAMfluorescein (FL) conjugate (BAM-FL, see Figure 2A) and subsequently opsonized tumor cells with tumor-nonspecific anti-FL antibodies (see Figure 2B). We further evaluated the efficiencies of phagocytosis and DC maturation ex vivo, and assessed antitumor protective effects and long-term antitumor memory induced by DC immunotherapy in vivo.

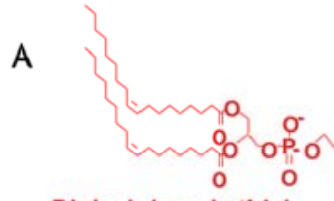

Dioleylphosphatidylethanolamine (DOPE)

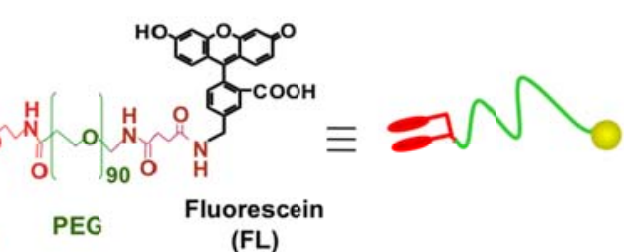

(FL)

B

Figure 2. Chemical structures and the approach used for opsonization. (A) The chemical structure of BAM-FL. (B) A schematic diagram of tumor cell-opsonization using a tumornonspecific anti-FL antibody strategy.

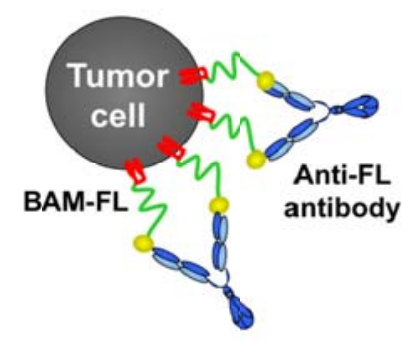




\section{Materials and methods}

\subsection{BAM reagents}

A cell membrane modifier, BAM [Dioleyl-phosphatidylethanolamine-poly(ethylene glycol)-succinyl-N-hydroxysuccinimidyl ester (DOPE-PEG-NHS)], was a gift from the NOF Company (Tokyo, Japan). Fluorescein-labeled BAM was prepared as previously described ${ }^{[25]}$. Briefly, $30 \mathrm{mM}$ 5-aminomethyl fluorescein hydrochloride solution $(30 \mu \mathrm{l})$ dissolved in dimethyl sulfoxide (DMSO) and $10 \mathrm{mM} \mathrm{BAM}(30 \mu \mathrm{l})$ solution dissolved in DMSO were mixed and incubated for $1 \mathrm{~h}$ at room temperature.

\subsection{Mice}

C57BL/6 (B6) mice (6- to 10-week-old females) were purchased from Charles River Laboratories Japan (Tokyo, Japan). Mice were bred and maintained in accordance with the guidelines of the Science Council of Japan.

\subsection{Antibodies and flow cytometry analysis}

Mouse anti-FL IgG and phycoerythrin (PE)-conjugated rat anti-Fc of mouse IgG were obtained from Neomarkers (Fremont, CA, USA) and Chemicon (Billerica, MA, USA), respectively. For DC immunostaining, PE-conjugated anti-mouse CD11c (a DC marker), PE-conjugated anti-mouse CD16/CD32 (FcyRIII/II), and PE-Cy5-conjugated anti-mouse CD86 (a mature DC marker) antibodies were purchased from eBioscience (San Diego, CA, USA). To block nonspecific binding of antibody to Fc $\gamma \mathrm{R}$ for DC and phagocytosis inhibition assays, anti-mouse CD16/CD32 antibody from BD Biosciences (Franklin Lakes, NJ, USA) was used.

Flow cytometry analysis was performed using a FACS Calibur with CellQuest software (BD Biosciences).

\subsection{Preparation of apoptotic tumor cells and opsonization with I gG}

Murine EL4 T-lymphoma cells were incubated in RPMI 1640 medium with 10\% FBS and $10 \mu \mathrm{g} / \mathrm{ml}$ mitomycin C (Wako Pure Chemical Industries; Tokyo, Japan) at $37^{\circ} \mathrm{C}$ in a $5 \% \mathrm{CO}_{2}$ incubator for $6 \mathrm{~h}$ to induce apoptotic cell death. After washing apoptotic tumor cells with PBS buffer twice, cells were incubated in PBS buffer with $10 \mathrm{mM}$ BAM-FL for $40 \mathrm{~min}$ at $37^{\circ} \mathrm{C}$, as previously described ${ }^{[25]}$. To prepare whole tumor cell-immune complexes (WTC-ICs), BAM-FL-conjugated cell membranes of tumor cells were further opsonized with mouse anti-FL IgG $(20 \mu \mathrm{g} / \mathrm{ml})$. The opsonization of WTCs was observed using a confocal laser-scanning microscope (CLSM; TCS-NT, Leica Microsystems, Wetzlar, Germany) by staining WTC-ICs with PE-conjugated rat anti-Fc domain antibody $(15 \mu \mathrm{g} / \mathrm{ml})$.

\subsection{Preparation of immature DCs derived from bone marrow cells}

C57BL/6 mice were used as a source of bone marrow-derived immature DCs, as previously described ${ }^{[28]}$, in accordance with a protocol approved by the Animal Care and Use Committee of the University of Tokyo (Permission for animal experiments in the Faculty of Engineering: No. K17-3). After red blood cells were lysed with red blood cell lysis buffer (Roche Applied Science; Indianapolis, IN, USA), bone marrow cells were seeded in 24-well plates at an initial concentration of $5 \times 10^{5}$ cells $/ \mathrm{ml}$ in $2 \mathrm{ml}$ RMPI 1640 medium (Nissui Pharmaceutical Co.; Tokyo, Japan) containing 10\% FBS (HyClone; Logan, UT, USA) and $10 \mathrm{ng} / \mathrm{ml}$ murine recombinant GM-CSF (Peprotech; Rocky Hill, NJ, USA) and were cultured at $37^{\circ} \mathrm{C}$ in a $5 \% \mathrm{CO}_{2}$ incubator. On days 2 and 4 of culture, $1 \mathrm{ml}$ culture supernatant was replaced with $1 \mathrm{ml}$ fresh RMPI 1640 medium with 10\% FBS and $10 \mathrm{ng} / \mathrm{ml} \mathrm{GM-CSF}$. Non-adherent and weakly adherent cells on day 6 of culture were harvested by strong pipetting and used as immature DCs in the following experiments.

\subsection{Phagocytosis tests and assessments of DC maturation}

Immature DCs were used for phagocytosis tests and assessments of DC maturation. EL4 cells were stained with $5 \mu \mathrm{M}$ green fluorescent dye, carboxyfluorescein diacetate succinimidyl ester (CFSE, Dojindo; Kumamoto, Japan), in PBS at 
$37^{\circ} \mathrm{C}$ for $30 \mathrm{~min}$. Apoptotic WTCs and WTC-ICs were prepared as mentioned above. For the phagocytosis tests, immature DCs $\left(10^{6}\right.$ cells $\left./ \mathrm{ml}\right)$ were co-cultured with CFSE-stained apoptotic WTCs or WTC-ICs at a 1:1 ratio of DCs-to-tumor cells in $1.5 \mathrm{ml}$ tubes with $500 \mu \mathrm{l}$ RPM 1640 medium containing $10 \mathrm{ng} / \mathrm{ml}$ murine recombinant GM-CSF at $37^{\circ} \mathrm{C}$ in a $5 \% \mathrm{CO}_{2}$ incubator. After co-culture for 1 or $24 \mathrm{~h}$, cells were harvested by centrifugation at $1000 \mathrm{rpm}$ for $3 \mathrm{~min}$, washed twice with PBS, and stained with PE-conjugated anti-mouse CD11c (a DC marker) antibody $(0.4 \mu \mathrm{g} / \mathrm{ml})$ at $4^{\circ} \mathrm{C}$ for $1 \mathrm{~h}$. For fluorescence microscopy analysis of phagocytosis, the uptake of CFSE-stained apoptotic WTCs-ICs by immature DCs was observed using a fluorescence microscope (IX81, Olympus; Tokyo, Japan).

The phagocytic activity of immature DCs was evaluated by flow cytometry by measuring cells positive for CFSE (green fluorescence) and PE (red fluorescence). The phagocytosis rate of immature DCs was determined by calculating the frequency of double-positive fluorescent cells among all red fluorescent cells.

To evaluate DC maturation, immature DCs $\left(10^{6} \mathrm{cells} / \mathrm{ml}\right)$ were pulsed with apoptotic WTC or WTC-ICs at a 1:1 ratio of DCs-to-tumor cells, and were co-cultured in $1.5 \mathrm{ml}$ tubes with $500 \mu \mathrm{l}$ RPMI 1640 medium containing $10 \mathrm{ng} / \mathrm{ml}$ murine recombinant GM-CSF at $37^{\circ} \mathrm{C}$ in a $5 \% \mathrm{CO}_{2}$ incubator for $24 \mathrm{~h}$. Then, cells were centrifuged at $1,000 \mathrm{rpm}$ for $3 \mathrm{~min}$, washed twice with PBS, and incubated in PBS containing anti-mouse CD16/CD32 (Fc $\gamma \mathrm{R} / \mathrm{III} / \mathrm{II})$ antibody $(1 \mu \mathrm{g} / \mathrm{ml})$ at $4^{\circ} \mathrm{C}$ for 30 min. Then, cells were stained with PE-Cy5-conjugated anti-mouse CD86 (a mature DC marker) antibody (0.4 $\mu \mathrm{g} / \mathrm{ml})$ and PE-conjugated anti-mouse CD11c antibody $(0.4 \mu \mathrm{g} / \mathrm{ml})$ at $4^{\circ} \mathrm{C}$ for $1 \mathrm{~h}$. Positively stained cells were quantitatively analyzed using flow cytometry as described above. The maturation rate of DCs was determined by calculating the frequency of double-positive cells for PE and PE-Cy5 staining among all PE-positive cells.

\subsection{Phagocytosis inhibition assays and DC maturation using an anti-Fc $\gamma \mathbf{R}$ antibody}

Immature DCs derived from bone marrow cells on day 6 of culture were used for inhibition assays of phagocytosis and DC maturation. Apoptotic WTCs and WTC-ICs were prepared as described above. For the inhibition assays of phagocytosis and DC maturation, immature DCs $\left(10^{6}\right.$ cells $\left./ \mathrm{ml}\right)$ were incubated with apoptotic WTCs or WTC-ICs at a 1:1 ratio of DCs-to-tumor cells in $1.5 \mathrm{ml}$ tubes with RPMI 1640 medium containing $10 \mathrm{ng} / \mathrm{ml}$ murine recombinant GM-CSF and $10 \mu \mathrm{g} / \mathrm{ml}$ anti-mouse $\mathrm{CD} 16 / \mathrm{CD} 32$ antibody at $37^{\circ} \mathrm{C}$ in a $5 \% \mathrm{CO}_{2}$ incubator. After $24 \mathrm{~h}$ incubation, cells were harvested by centrifugation at 1,000 rpm for $3 \mathrm{~min}$, and then were washed twice with PBS. Phagocytic capacity was evaluated by staining DCs with PE-conjugated anti-mouse CD11c antibody. Additionally, DC maturation was evaluated by staining DCs with PE-Cy5-conjugated anti-mouse CD86 antibody and analyzing cells by flow cytometry as described above.

\subsection{Evaluation of in vivo antitumor protection effects and long-term antitumor memory}

WTC-IC-pulsed mature DCs $\left(1 \times 10^{6}\right.$ cells/100 $\mu$ PBS per mouse) were administered by intraperitoneal injection in mice, and the same immunization was repeated once 1 week later. Alternatively, as control experiments, WTC-pulsed DCs or PBS alone was injected into mice. Each treatment group included six or seven mice. Then, 7 days after the final vaccination, mice were challenged subcutaneously with EL4 cells $\left(4 \times 10^{3}\right.$ cells $/ 50 \mu \mathrm{PBS} /$ mouse $)$ and were evaluated daily until death.

Long-term antitumor memory was also investigated by challenging mice with EL4 cells $\left(8 \times 10^{3}\right.$ cells $\left./ 50 \mu \mathrm{PBS} / \mathrm{mouse}\right)$ subcutaneously; mice that had survived for 90 days $(n=4)$ in the group of mice treated with WTC-ICs-pulsed DCs were used in this experiment. All animal experiments were performed in accordance with a protocol approved by the Animal Care and Use Committee of the University of Tokyo (Permission for animal experiments in the Faculty of Engineering: No. K17-3). 


\subsection{Statistical analyses}

Statistically significant differences were detected using Student's $t$-test for the phagocytosis inhibition and DC maturation assays, or with the log-rank test for mouse survival curves. Differences were considered to be significant at a threshold of $P<.05$.

\section{Results}

\subsection{Opsonization of tumor cells with a tumor-conjugated nonspecific antibody using BAM}

EL4 cells were incubated with BAM-FL, and then with mouse anti-FL IgG. To confirm the opsonization of cells with mouse anti-FL IgG, cells were stained with PE-conjugated anti-mouse Fc antibody. The red fluorescence of PEconjugated anti- mouse Fc antibody was detected on the cell membrane (see Figure 3), indicating that the anti-FL antibody successfully bound to BAM-FL anchored-cell membranes and that the $\mathrm{Fc}$ of the antibody was oriented away from the cell membrane.

Figure 3. Confocal laser-scanning microscopy observation of mouse anti-FL antibody staining of BAM-FL-conjugated tumor cell membranes. Images show BAM-FL-conjugated tumor cell membranes stained with mouse anti-FL antibody and PE-conjugated rat anti-Fc of mouse IgG.

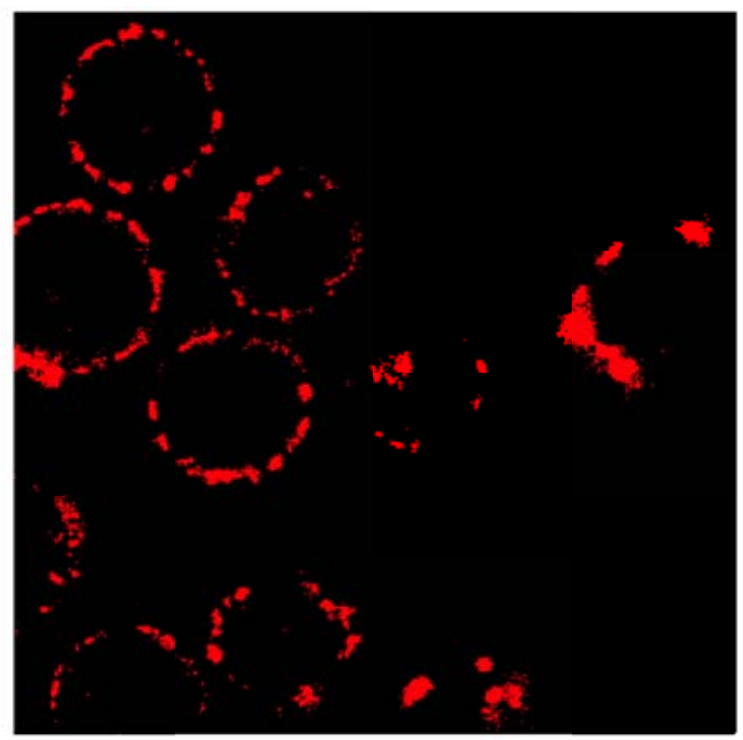

\subsection{Phagocytosis, DC maturation, and DC inhibition assays}

First, fluorescent microscopy analysis was performed to confirm the uptake of tumor cell-derived apoptotic bodies by DCs. The DC outer membranes were stained red because of the binding of PE-conjugated anti-CD11c antibody. By contrast, WTC-ICs were stained green with CFSE. Several red-stained DCs were observed that had incorporated green-stained apoptotic bodies (they appeared yellow because of red and green double-positivity) inside cells (see Figure $4 \mathrm{~A}$, merged image).

Flow cytometry was performed to evaluate phagocytic activity and DC maturation. While the percentage of tumorincorporated DCs was $62 \%$ of total DCs when DCs were co-cultured with WTCs for $24 \mathrm{~h}$, it increased to $83 \%$ when DCs were co-cultured with WTC-ICs and was reduced to $66 \%$ in the presence of anti-CD16/CD32 (Fc $\gamma$ RIII/II) antibody (see Figure 4B). Flow cytometry analysis also showed that the percentage of DCs that highly expressed CD86 was $12 \%$ of total DCs when immature DCs were co-cultured with WTCs, but was $22 \%$ when co-cultured with WTC-ICs and was reduced to $11 \%$ in the presence of anti-CD16/CD32 antibody (see Figure 4C). 

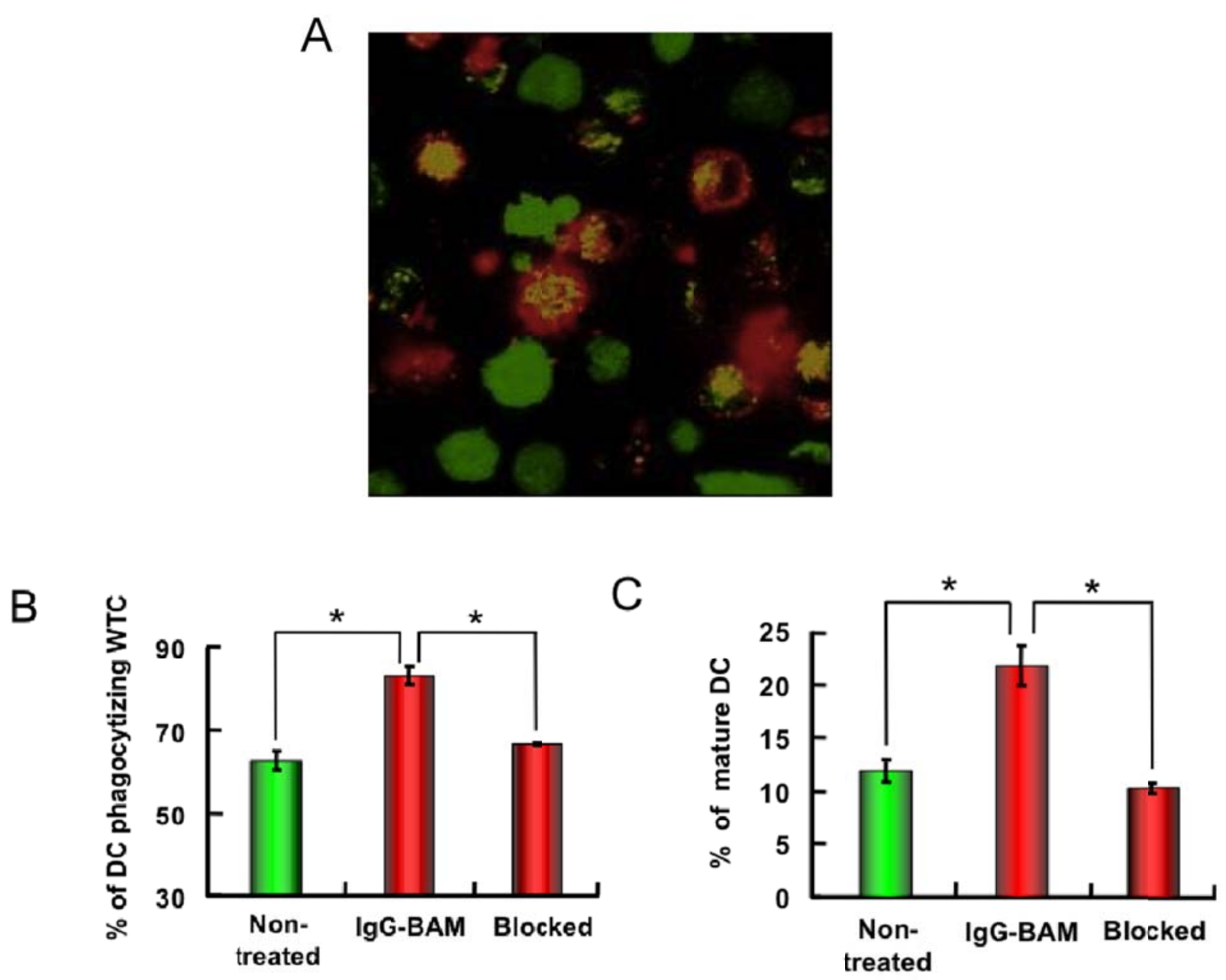

Figure 4. The effects of opsonization on phagocytosis and DC maturation. (A) Fluorescence microscopy observation of DCs (red cells) phagocytizing apoptotic bodies of WTC-ICs (green cells). The effects of cell-opsonization with a tumor-nonspecific antibody were analyzed for the following parameters: (B) the phagocytic activity of immature DCs and (C) DC maturation efficiency with or without the addition of anti-CD16/CD32 (Fc $\gamma \mathrm{R}$ III/II) antibody. Immature DC cells were co-cultured for $24 \mathrm{~h}$ at a 1:1 ratio with either apoptotic WTCs (nontreated), apoptotic WTC-ICs (IgG-BAM), or apoptotic WTC-ICs in the presence of anti-Fc $\gamma$ R antibody (blocked). The percentages of DCs phagocytizing WTCs and matured DCs were analyzed by flow cytometry using PE-conjugated anti-mouse CD11c (a DC marker) antibody and PE-Cy5-conjugated anti-mouse CD86 (a mature DC marker) antibody, respectively. Data are presented as means $\pm \mathrm{SD}$ of five independent experiments; ${ }^{*} P<.05$ (Student's $t$-test).

\subsection{DCs co-cultured with WTC-I Cs elicited potent antitumor immunity and long-term memory in vivo}

To test whether antitumor effects could be elicited by DCs that incorporated antibody-opsonized tumor cells, B6 mice were immunized intraperitoneally twice with DCs that were co-cultured for $24 \mathrm{~h}$ with either WTCs or WTC-ICs. Then, 7 days after the final vaccination, mice were injected subcutaneously with EL4 cells. We found that four of seven mice (57\%) that were immunized with DCs co-cultured with WTC-ICs survived for 90 days. By contrast, four mice immunized with DCs co-cultured with WTCs showed slightly stronger antitumor immunity compared with mice immunized with PBS (naive), but exhibited no significant protection against the inoculated EL4 cells (see Figure 5A). 
To investigate the long-term maintenance of these antitumor effects, EL4 cells were again subcutaneously challenged into four mice after survival for 90 days. We found that two of four mice (50\%) survived for another 90 days (see Figure 5B).

A

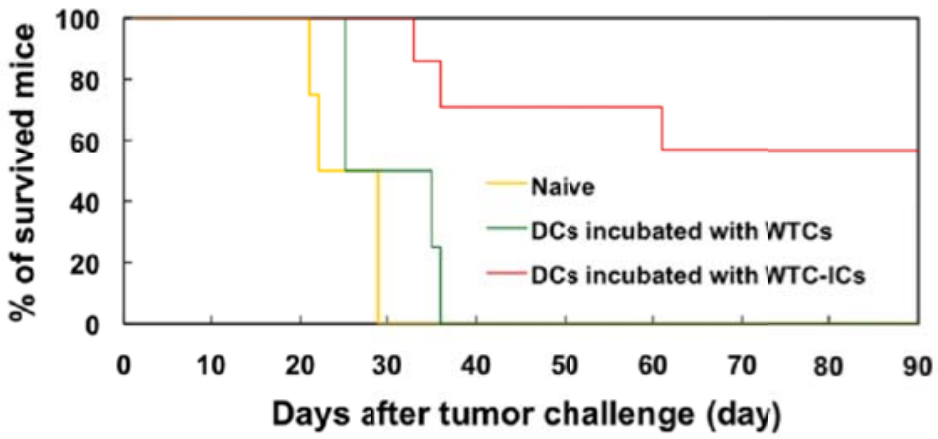

$\mathrm{B}$

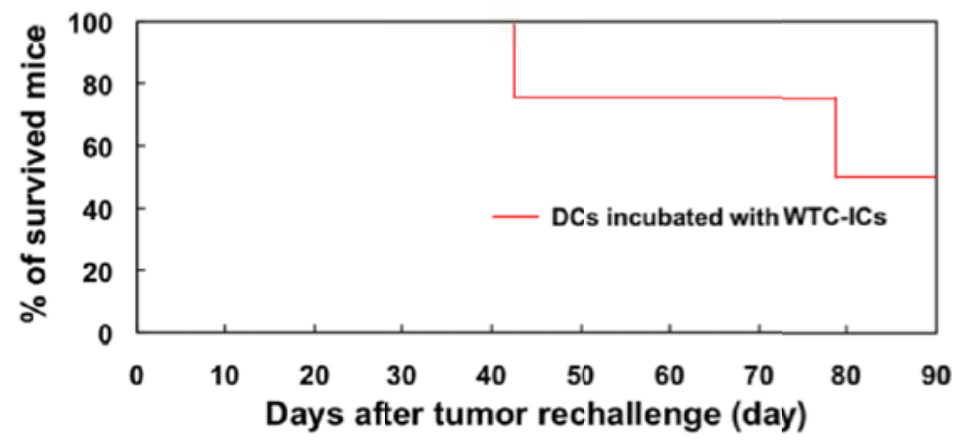

Figure 5. The therapeutic effects of tumor vaccination with DCs. (A) B6 mice were immunized with either PBS (naive), DCs incubated with WTCs, or DCs incubated with WTC-ICs. Day 0 indicates the day of tumor cell challenge. Survival curves of the treatment groups are shown. Each treatment group included four or seven mice. Statistical analysis was performed using the log-rank test. $P$-values were $P=.197$ and $P=.000799$ for DCs incubated with WTCs or WTC-ICs, respectively, vs. naive mice. (B) Mice were rechallenged with EL4 cells after surviving for 90 days $(n=4$ mice that were treated with WTC-ICs-pulsed DCs). Day 0 indicates the day of tumor cell rechallenge. Survival curve of tumorrechallenged mice is shown.

\section{Discussion}

The control of antibody orientation on the tumor cell membrane is an important issue in efforts to promote ADPC because the Fc of an antibody is recognized by the Fc $\gamma \mathrm{R}$ of immature DCs. Although the direct chemical conjugation of an antibody to cell surface proteins is one method for the opsonization of tumor cell membranes ${ }^{[23]}$, it is difficult to control antibody orientation because chemical conjugation is usually an amino acid-specific reaction and several reactive amino acids can exist on the surface of an antibody. Furthermore, the chemical conjugation of tumor membrane proteins with an antibody can alter the chemical structure and properties of cell surface antigens. Such alterations in the chemical structure and properties of antigens can lead to reductions in the repertoire of antigenic epitopes for presentation to antitumor effector and helper immune cells.

Previously, we successfully achieved the direct opsonization of WTCs using BAM-antibody or BAM-Fc domain conjugates and enhanced the phagocytic ratio of WTCs by immature DCs ${ }^{[28,29]}$. These BAM-mediated opsonization methods have the distinct advantage of enhancing phagocytic activity without altering any chemical structures of the cell surface antigens. Although optimizing the chemical conjugation conditions of BAM to antibody can minimize interference 
in the interactions between the Fc domain of an antibody on tumor cells and the FcyR on immature DCs, it remained difficult to specifically control the conjugation site of an antibody with BAM, resulting in insufficient spatial control of $\mathrm{Fc}$ domain orientation toward Fc $\gamma$ R. Site-specific BAM conjugation to the C-terminus of the Fc domain by sortase A-mediated enzymatic ligation is one way to solve this problem. However, the yields of soluble intact Fc domain produced by mammalian cell culture and of $\mathrm{Fc}$ domain-BAM conjugation reaction are not sufficient for widespread clinical application. To overcome these challenges, we aimed to develop a more general and simple method to promote ADCP of DCs using BAM-hapten conjugates and anti-hapten antibodies. We evaluated the efficiencies of phagocytosis and DC maturation ex vivo and tested antitumor protection effects and long-term antitumor memory induced by DC immunotherapy in vivo.

We found that mouse anti-FL antibody bound to BAM-FL on the cell membrane, which we could successfully stain with PE-conjugated anti-mouse Fc domain antibody. This finding indicates that tumor cells can be opsonized with tumor-nonspecific anti-hapten antibodies and that the $\mathrm{Fc}$ of the antibody was directed away from the tumor cell membrane. Because the chemical conjugation reaction between BAM and FL is site-specific and has a high yield, and the antibody does not need to be modified, this tumor opsonization method might represent a promising method for economically and efficiently supplying WTC-ICs for clinical use.

Tumor cells opsonized with specific antibodies against tumor-specific antigens have been reported to promote the phagocytic activity and maturation of DCs ${ }^{[17]}$. Herein, we tested whether FL-BAM-generated tumor-nonspecific antibody-opsonized tumor cells could be effectively phagocytized by DCs and promote the maturation of DCs. In merged fluorescence images, several red-stained DCs were found to have incorporated green-stained apoptotic bodies of WTC-ICs (yellow). Consequently, fluorescent images clearly confirmed that DCs phagocytized WTC-ICs at a high rate. When immature DCs were co-cultured with WTC-ICs, the phagocytosis and DC maturation rates were enhanced by $\sim 20 \%$ and $\sim 10 \%$, respectively, compared with DCs co-cultured with WTCs. These enhancements could be blocked by the addition of anti-CD16/CD32 (FcyRIII/II) antibody. These findings indicate that a tumor-nonspecific antibody could also promote ADCP by immature DCs and DC maturation through interactions between the Fc of an antibody and the Fc $\gamma \mathrm{R}$ of DCs, similar to the mechanism of tumor-specific antibodies ${ }^{[17,21-24]}$.

We investigated the antitumor effects of mature DCs co-cultured for $24 \mathrm{~h}$ with either WTCs or WTC-ICs by immunizing B6 mice twice with mature DCs. Mice immunized with PBS could not survive for more than 30 days after tumor cell challenge. By contrast, mice immunized with DCs co-cultured with WTCs showed slightly stronger antitumor immunity than did mice immunized with PBS, but no significant protective effects were observed. By contrast, mice immunized with DCs co-cultured with WTC-ICs showed robust antitumor immunity and four of seven mice (57\%) survived for 90 days. These four mice that survived for 90 days were subjected to assessments of long-term antitumor memory, and two of four mice survived for another 90 days after rechallenge with tumor cells. These findings show that DCs co-cultured with tumor-nonspecific antibody-opsonized tumor cells could enhance in vivo antitumor responses and induce long-term antitumor memory in vivo. Because DCs can efficiently present tumor cell antigens, which can be phagocytized through a Fc $\gamma$ R-mediated pathway, on both MHC I and II ${ }^{[30]}$, DCs can subsequently activate not only CTL but also NK cells ${ }^{[16,23]}$. Therefore, ADCP using BAM-hapten conjugates and anti-hapten antibodies might induce effective antitumor responses by mature DCs and elicit long-term antitumor memory.

\section{Conclusion}

In the present study, we modified apoptotic tumor cell surfaces with BAM-FL conjugates and opsonized them with an anti-FL antibody. By co-culturing DCs with antibody-opsonized tumor cells, the phagocytosis and DC maturation rates were enhanced by $\sim 20 \%$ and $\sim 10 \%$, respectively, compared with co-culture with non-opsonized tumor cells. Furthermore, the administration of tumor cell antigen-loaded mature DCs to mice resulted in enhanced tumor rejection and long-term survival. The advantages of this method are that tumor-specific antibodies are not required and DCs can present 
uncharacterized antigens on MHC molecules by uptake of the antigenic contents of WTCs. Therefore, this method might be applicable to various types of tumors for which the tumor-specific antigens have not yet been identified.

Generally, immature DCs, which present tumor antigen to naive T cells without co-stimulation by CD86, have some possibility of leading to either the deletion of $\mathrm{T}$ cells or the generation of inducible regulatory $\mathrm{T}$ cells and inducing tolerance against tumor ${ }^{[3]}$. However further enhancement of DCs maturation by cytokines or separation and selection of matured DCs after co-culture of DCs with antibody-opsonized tumor cells could improve the clinical efficacy of DC immunotherapy.

\section{Acknowledgements}

We thank the NOF Corporation (Tokyo, Japan) for generously providing DOPE-PEG-NHS. This research was supported by Japan MEXT (Ministry of Education, Culture, Sports, Science and Technology), a Grant-in-Aid for Exploratory Research (15656208), and the Center for NanoBio Integration at The University of Tokyo.

\section{References}

[1] Lu W, Wu X, Lu Y, et al. Therapeutic dendritic-cell vaccine for simian AIDS. Nat Med. 2003; 9: 27-32. PMid:12496959. http://dx.doi.org/10.1038/nm806

[2] Figdor CG, de Vries IJ, Lesterhuis WJ, et al. Dendritic cell immunotherapy: Mapping the way. Nat Med. 2004; 10: 475-80. PMid:15122249. http://dx.doi.org/10.1038/nm1039

[3] Banchereau J, Palucka AK. Dendritic cells as therapeutic vaccines against cancer. Nat Rev Immunol. 2005; 5: 296-306. PMid:15803149. http://dx.doi.org/10.1038/nri1592

[4] Steinman RM, Banchereau J. Taking dendritic cells into medicine. Nature. 2007; 449: 419-26. PMid:17898760. http://dx.doi.org/10.1038/nature06175

[5] Vanneman M, Dranoff G. Combining immunotherapy and targeted therapies in cancer treatment. Nat Rev Cancer. 2012; 12: 237-51. PMid:22437869. http://dx.doi.org/10.1038/nrc3237

[6] Svane IM, Soot ML, Buus S, et al. Clinical application of dendritic cells in cancer vaccination therapy. APMIS. 2003; 111 : 818-34. PMid:12974783. http://dx.doi.org/10.1034/j.1600-0463.2003.11107813.x

[7] Brossart P, Wirths S, Stuhler G, et al. Induction of cytotoxic T-lymphocyte responses in vivo after vaccinations with peptide-pulsed dendritic cells. Blood. 2000; 96: 3102-8.

[8] Machy P, Serre K, Leserman L. Class I-restricted presentation of exogenous antigen acquired by Fcy receptor-mediated endocytosis is regulated in dendritic cells. Eur J Immunol. 2000; 30: 848-57. http://dx.doi.org/10.1002/1521-4141(200003)30:3<848::AID-IMMU848>3.0.CO;2-Q

[9] den-Haan JMM, Bevan MJ. Constitutive versus activation-dependent cross-presentation of immune complexes by CD8+ and CD8- dendritic cells in vivo. J Exp Med. 2002; 196: 17-27. http://dx.doi.org/10.1084/jem.20020295

[10] Kalergis AM, Ravetch JV. Inducing tumor immunity through the selective engagement of activating Fc receptors on dendritic cells. J Exp Med. 2002; 195: 1653-9. http://dx.doi.org/10.1084/jem.20020338

[11] Thumann P, Moc I, Humrich J, et al. Antigen loading of dendritic cells with whole tumor cell preparations. J Immunol Methods. 2003; 277: 1-16. http://dx.doi.org/10.1016/S0022-1759(03)00102-9

[12] Kugler A, Stuhler G, Walden P, et al. Regression of human metastatic renal cell carcinoma after vaccination with tumor cell-dendritic cell hybrids. Nat Med. 2000; 6: 332-6. PMid:10700237. http://dx.doi.org/10.1038/73193

[13] Galea-Lauri J, Darling D, Mufti G, et al. Eliciting cytotoxic T lymphocytes against acute myeloid leukemia-derived antigens: Evaluation of dendritic cell-leukemia cell hybrids and other antigen-loading strategies for dendritic cell-based vaccination. Cancer Immunol Immunother. 2002; 51: 299-310. PMid:12111118. http://dx.doi.org/10.1007/s00262-002-0284-4

[14] Phan V, Errington F, Cheong SC, et al. A new genetic method to generate and isolate small, short-lived but highly potent dendritic cell-tumor cell hybrid vaccines. Nat Med. 2003; 9: 1215-9. http://dx.doi.org/10.1038/nm923

[15] Kotera Y, Shimizu K, Mulé JJ. Comparative analysis of necrotic and apoptotic tumor cells as a source of antigen(s) in dendritic cell-based immunization. Cancer Res. 2001; 61: 8105-9.

[16] Schnurr M, Scholz C, Rothenfusser S, et al. Apoptotic pancreatic tumor cells are superior to cell lysates in promoting cross-priming of cytotoxic T cells and activate NK and $\gamma \delta$ T cells. Cancer Res. 2002; 62: 2347-52. 
[17] Chen Z, Moyana T, Saxena A, et al. Efficient antitumor immunity derived from maturation of dendritic cells that had phagocytosed apoptotic/necrotic tumor cells. Int J Cancer. 2001; 93: 539-48. PMid:11477558. http://dx.doi.org/10.1002/ijc.1365

[18] Paczesny S, Beranger S, Salzmann JL, et al. Protection of mice against leukemia after vaccination with bone marrow-derived dendritic cells loaded with apoptotic leukemia cells. Cancer Res. 2001; 61: 2386-9.

[19] Nestle FO, Farkas A, Conrad C. Dendritic-cell-based therapeutic vaccination against cancer. Curr Opin Immunol. 2005; 17: 163-9. PMid:15766676. PMid:15766676. http://dx.doi.org/10.1016/j.coi.2005.02.003

[20] Janikashvili N, Larmonier N, Katsanis E. Personal dendritic cell-based tumor immunotherapy. Immunotherapy. $2010 ; 2: 57-68$. PMid:20161666. http://dx.doi.org/ 10.2217/imt.09.78

[21] Selenko N, Maidic O, Draxier S, et al. CD20 antibody (C2B8)-induced apotosis of lymphoma cells promotes phagocytosis by dendritic cells and cross-priming CD8+ cytotoxic T cells. Leukemia. 2001; 15: 1619-26. PMid:11587221. http://dx.doi.org/10.1038/sj.leu.2402226

[22] Dhodapkar KM, Krasovsky J, Williamson B, et al. Antitumor monoclonal antibodies enhance cross-presentation of cellular antigens and the generation of myeloma-specific killer T cells by dendritic cells. J Exp Med. 2002; 195: 125-33. PMid:11781371. http://dx.doi.org/10.1084/jem.20011097

[23] Akiyama K, Ebihara S, Yada A, et al. Targeting apoptotic tumor cells to Fc $\gamma \mathrm{R}$ provides efficient and versatile vaccination against tumors by dendritic cells. J Immunol. 2003; 170: 1641-8. PMid:12574326. http://dx.doi.org/10.4049/jimmunol.170.4.1641

[24] Groh V, Li YQ, Cioca D, et al. Efficient cross-priming of tumor antigen-specific T cells by dendritic cells sensitized with diverse anti-MICA opsonized tumor cells. Proc Natl Acad Sci USA. 2005; 102: 6461-6. PMid:15824323. http://dx.doi.org/10.1073/pnas.0501953102

[25] Kato K, Itoh C, Yasukouchi T, et al. Rapid protein anchoring into the membranes of mammalian cells using oleyl chain and poly(ethylene glycol) derivatives. Biotechnol Prog. 2004; 20: 897-904. PMid:15176897. http://dx.doi.org/10.1021/bp0342093

[26] Chung HA, Kato K, Itoh C, et al. Casual cell surface remodeling using biocompatible lipid-poly(ethylene glycol)(n): development of stealth cells and monitoring of cell membrane behavior in serum-supplemented condition. J Biomed Mater Res. 2004; 70A: 179-85. http://dx.doi.org/10.1002/jbm.a.20117

[27] Chung HA, Tajima K, Kato K, et al. Modulating the actions of NK cell-mediated cytotoxicity using lipid-PEG(n) and inhibitory receptor-specific antagonistic peptide conjugates. Biotechnol Prog. 2005; 21: 1226-30. PMid:16080706. http://dx.doi.org/10.1021/bp049646b

[28] Tomita U, Yamaguchi S, Sugimoto Y, et al. Poly(ethylene glycol)-lipid-conjugated antibodies enhance dendritic cell phagocytosis of apoptotic cancer cells. Pharmaceuticals. 2012; 5: 405-16. PMid:24281554. http://dx.doi.org/10.1021/bp049646b

[29] Tomita U, Yamaguchi S, Maeda Y, et al. Protein cell-surface display through in situ enzymatic modification of proteins with a poly(Ethylene glycol)-lipid. Biotechnol Bioeng. 2013; 110: 2785-9. PMid:23592269. http://dx.doi.org/10.1002/bit.24933

[30] Amigorena S. Fc $\gamma$ receptors and cross-presentation in dendritic cells. J Exp Med. 2002; 195: F1-3. http://dx.doi.org/10.1084/jem.20011925 